Original Research Paper

\title{
Effect of the Maximum Density Ratio Between Liquid and Vapor on Cavitating Simulation
}

\author{
Guangjian Zhang, Weidong Shi, Ling Zhou and Desheng Zhang \\ Research Center of Fluid Machinery Engineering and Technology, Jiangsu University, Zhenjiang, China
}

Article history

Received: 02-03-2015

Revised: 18-03-2015

Accepted: 06-04-2015

Corresponding Author:

Ling Zhou

Research Center of Fluid

Machinery Engineering and

Technology, Jiangsu University,

Zhenjiang, China

Email: lingzhoo@hotmail.com

\begin{abstract}
The Filter-Based Model (FBM), which was built into CFX through CFX Expression Language (CEL) and a homogeneous cavitation model were employed to simulate cavitating flow around a 2D Clark-y hydrofoil. The effect of a maximum density ratio between liquid and vapor on sheet and cloud cavitating simulation was investigated. The results show that the maximum density ratio has a significant impact on cavitating simulation. The predicted cavitation with default value 1000 is underestimated compared with experiment. With the increasing of maximum density ratio, the interaction interface between liquid and vapor becomes unstable, accompanying the intermittent shedding of small-scale cavities. The cavity length and vapor volume fraction also increase. When the maximum density is increased to some degree, its effect on cavitation flow calculation becomes unobvious. A smaller maximum density ratio can ensure numerical stability but the result predicted with true density ratio is more accurate, so 20000 is recommended as the value of maximum density ratio in cavitation model to reach an optimum between accuracy and convergence.
\end{abstract}

Keywords: Cavitation Model, Maximum Density Ratio, Filter-Based Model, Cavity Shape

\section{Introduction}

Cavitation is a complex phenomenon involving phase transition, unsteadiness and multi-scale turbulence. It is well known that strong instability of cloud cavitation often leads to serious problems in hydraulic machinery such as noise, vibration and erosion. There have been a number of recent experimental and numerical studies examining the complex multiphase structures in developed cavitating flows (Leroux et al., 2005; Wang et al., 2001; Zhou and Wang, 2008; Ducoin et al., 2012), which show that the reentrant flow plays a major role on instability of cavitating flow. Especially, the cavitation model is a critical method determining the accuracy of numerical simulation. Esfahanian et al. (2012) applied a barotropic state equation that links the density variations to the local static pressure to solve the mixture density in the cavitating flow and this method is famous as the cavitation model based on state equation. The other popular approach to simulate cavitating flows is called as the cavitation model based on transport equation, which solves an additional transport equation for either the mass or volume fraction of vapor, with appropriate source/sink term(s) to regulate the mass transfer between the liquid and vapor phases. Different source/sink terms have been proposed, including the popular models presented in (Zwart et al., 2004; Singhal et al., 2002; Kunz et al., 1998). Huang et al. (2009) evaluated the above three kind of transport-based cavitation models in the same software and showed that all three different cavitation models can predict successively the main characteristics of cavitation and the detailed process of the cavitation development has small differences. Morgut et al. (2011) indicated these models share the common feature of employing empirical coefficients, to tune the models of condensation and evaporation processes that can influence the accuracy and stability of the numerical predictions. Ji et al. (2013) also pointed out the effect of empirical coefficients in simulating cavitating flow around the 3D twisted hydrofoil. Huang et al. (2010) showed that the assumed bubble radius and volume fraction of non-condensable gas in cavitation models have significant effects on numerical simulation. 
The filter-based turbulence model and a homogeneous cavitation model were employed to simulate cavitating flow around a 2D Clark-y hydrofoil in ANSYS CFX. The effect of a maximum density ratio between liquid and vapor on sheet and cloud cavitating simulation was investigated.

\section{Numerical Method}

\section{Governing Equations}

The vapor/liquid two-phase mixture model assumes the fluid to be homogeneous, so the multiphase fluid components are assumed to share the same velocity and pressure. The continuity and momentum equations for the mixture flow are Equation 1 and 2:

$$
\frac{\partial \rho_{m}}{\partial t}+\frac{\partial\left(\rho_{m} u_{j}\right)}{\partial x_{j}}=0
$$

$$
\begin{gathered}
\frac{\partial\left(\rho_{m} u_{i}\right)}{\partial t} \frac{\partial\left(\rho_{m} u_{i} u_{j}\right)}{\partial x_{j}}=-\frac{\partial p}{\partial x_{i}}+\frac{\partial}{\partial x_{j}} \\
{\left[\left(\mu_{m}+\mu_{t}\right)\left(\frac{\partial u_{i}}{\partial x_{j}}+\frac{\partial u_{j}}{\partial x_{i}}-\frac{2}{3} \frac{\partial u_{k}}{\partial x_{k}} \delta_{i j}\right)\right]}
\end{gathered}
$$

Where:

$\rho_{m}=$ the density of mixture flow

$u_{i}=$ the instant velocity in the $i$ direction

$p=$ the mixture pressure

$\mu_{m}=$ the mixture laminar viscosity

$\mu_{t}=$ the turbulent eddy viscosity, which is obtained by the following turbulence model.

The mixture density $\rho_{\mathrm{m}}$ is defined as Equation 3:

$$
\rho_{m}=\rho_{l} \alpha_{l}+\rho_{v}\left(1-\alpha_{l}\right)
$$

where, $\rho$ and $\alpha$ are the density and the volume fraction. The subscript $v$ and $l$ refer to the vapor and liquid components respectively.

\section{Filter-Based Turbulence Model}

FBM is a hybrid model proposed by Johansen et al. (2004) based on the standard $k-\varepsilon$ model, which blends the RANS and LES methods. The turbulence with different scales can be solved by different methods via applying the filter function, which improves the prediction accuracy of multi-scale turbulent flow. Considering the advantage of RNG $k-\varepsilon$ model compared to the standard one, the FBM employed in the present study will be based on the RNG $k-\varepsilon$ model. The detailed equations are as follows Equation 4 and 5:

$$
\begin{aligned}
& \frac{\partial\left(\rho_{m} k\right)}{\partial t}+\frac{\partial\left(\rho_{m} u_{i} k\right)}{\partial x_{i}}=\frac{\partial}{\partial x_{j}}\left[\left(\mu_{m}+\frac{\mu_{t}}{\sigma_{k}}\right) \frac{\partial k}{\partial x_{j}}\right]+G_{k}+\rho_{m} \varepsilon \\
& \frac{\partial\left(\rho_{m} \varepsilon\right)}{\partial t}+\frac{\partial\left(\rho_{m} u_{i} \varepsilon\right)}{\partial x_{i}}=\frac{\partial}{\partial x_{j}}\left[\left(\mu_{m}+\frac{\mu_{t}}{\sigma_{\varepsilon}}\right) \frac{\partial \varepsilon}{\partial x_{j}}\right] \\
& +C_{\varepsilon 1} G_{k} \frac{\varepsilon}{k}-C_{\varepsilon 2} \rho_{m} \frac{\varepsilon^{2}}{k}
\end{aligned}
$$

where, $k$ and $\varepsilon$ are turbulent kinetic energy and turbulent dissipation rate respectively, $G_{k}$ is turbulence production rate, $C_{\varepsilon 1}=1.42, C_{\varepsilon 2}=1.68, \sigma_{k}=\sigma_{\varepsilon}=0.7176$.

The eddy viscosity is defined as Equation 6:

$$
\mu_{t}=\rho_{m} C_{\mu} \frac{k^{2}}{\varepsilon} f_{F B M}
$$

where, $C_{\mu}=0.085, f_{F B M}$ is the filter function, which depends on the ratio of filter scale $\Delta$ and turbulence scale $l_{R A N S}=k^{3 / 2} / \varepsilon$. The detailed Equation 7 is as follows:

$$
f_{F B M}=\operatorname{Min}\left(1, C \frac{\Delta}{k^{3 / 2} / \varepsilon}\right), C_{3}=1.0
$$

When $l_{R A N S}<\Delta$, the filter function returns the value of 1.0 , i.e., the RNG $k-\varepsilon$ model is recovered. When $l_{R A N S}>\Delta$, the filter function returns the identical form as the oneequation LES model.

\section{Cavitation Model}

Cavitation model describes the mass transfer between liquid and vapor. The present paper employed the ZwartGerber-Belamri cavitation model derived from a simplified Rayleigh-Plesset equation which neglects the second-order derivation of the bubble radius. The vapor density is clipped in a user-controlled fashion by the maximum density ratio $\rho_{l} / \rho_{v, c l i p}$ to control the numerical stability. The maximum density ratio is used to clip the vapor density for all terms except for the cavitation source term, which uses the true density specified as the material property.

The vapor volume fraction is governed by the following Equation 8 and 9:

$$
\begin{aligned}
& \frac{\partial \rho_{v, \text { clip }} \alpha_{v}}{\partial t}+\frac{\partial\left(\rho_{v, \text { clip }} \alpha_{v} u_{j}\right)}{\partial x_{j}}=\dot{m} \\
& \dot{m}=F_{e} \frac{3 \alpha_{m u c}\left(1-\alpha_{v}\right) \rho_{v}}{R_{B}} \sqrt{\frac{2}{3} \frac{\max \left(p_{v}-p, 0\right)}{\rho_{l}}} \\
& -F_{c} \frac{3 \alpha_{v} \rho_{v}}{R_{B}} \sqrt{\frac{2}{3} \frac{\max \left(p-p_{v}, 0\right)}{\rho_{l}}}
\end{aligned}
$$


Where:

$\dot{m}=$ The cavitation source term, which controls the mass transfer rate between liquid and vapor

$\rho_{v, c l i p}=$ The clipped vapor density

$p_{v} \quad=$ The saturated liquid vapor pressure

$F_{e}$ and $F_{c}=$ The empirical coefficients for vaporization and condensation processes.

$\alpha_{\text {muc }}=$ The non-condensable vapor fraction

$R_{B} \quad=$ The bubble size.

These empirical constants are set as $F_{e}=50, F_{c}=$ $0.01, \alpha_{\text {nuc }}=5 \times 10^{-4}, R_{B}=10^{-6} \mathrm{~m}$.

\section{Numerical Setup and Description}

The computational domain and boundary conditions are as shown in Fig. 1. The Clark-y hydrofoil is fixed in the center of a water channel with the angle of attack $\alpha=8$ degrees and the chord length $\mathrm{c}=70 \mathrm{~mm}$. The condition of no-slip wall is imposed on the hydrofoil surface and side surfaces of the tunnel. The inlet velocity is set to be $U_{\text {in }}=10 \mathrm{~m} \mathrm{sec}^{-1}$ corresponding to a Reynolds number $R e=7 \times 10^{5}$. The outlet pressure is determined by the cavitation number. The number of total grid elements is 54326 as shown in Fig. 2. The variation of $y+$ along the hydrofoil surface is plotted as shown in Fig. 3. The range of $\mathrm{y}+$ value is from 40 to 100 (except for the leading edge surface) which can meet the requirement of the Scalable Wall Function in CFX.

The unsteady cavitating flow simulations were started from a steady non-cavitation flow result. It used the time step $\Delta t=0.1 \mathrm{~ms}$ for the revolution calculation. During the unsteady calculation, the convergence in each physical time step was achieved in 4-10 iterations when the Root Mean Square (RMS) residual dropped below $10^{-5}$.

The pressure coefficient distribution $\left(-C_{p}=\left(P_{\text {out }}{ }^{-}\right.\right.$ $\left.P) /\left(0.5 \rho_{l} U_{\text {in }}{ }^{2}\right)\right)$ predicted via the present numerical model was compared with the experiment results (Wang et al., 2001) in non-cavitation. As shown in Fig. 4, a good agreement of the pressure coefficient distribution was found between the experiment and simulation results demonstrating the numerical method is feasible in the present work.

\section{Results and Discussion}

\section{Effect of Maximum Density Ratio on Simulating Sheet Cavitation}

In order to ensure the simulation is stable, a maximum density ratio $\rho_{l} / \rho_{v}$, clip is introduced to control the vapor density.
Substituting $\rho_{v, \text { clip }}=\frac{\rho_{l} / \rho_{v}}{\rho_{l} / \rho_{v, \text { clip }}} \rho_{v}$ into Equation 8, it can be written as:

$$
\frac{\partial \rho_{v} \alpha_{v}}{\partial t}+\frac{\partial\left(\rho_{v} \alpha_{v} u_{j}\right)}{\partial x_{j}}=\frac{\rho_{l} / \rho_{v, c l i p}}{\rho_{l} / \rho_{v}} \dot{m}
$$

As expressing in Equation 10, the cavitation mass transfer rate (the right term) has a strong relation with the maximum density ratio.

As described in Wang et al. (2001), the flow regime around the hydrofoil is sheet cavitation when cavitation number $\sigma=1.4$. The sheet cavity attaches on the suction side of hydrofoil from the near leading edge to $0.4 \mathrm{c}$. The main part of the cavity is stable while the rear region of the sheet is unsteady rolling up into a series of bubbles that shed intermittently. The simulation was implemented to study the effect of maximum density ratio with three specific values, that is 1000 (the default value in CFX), 20000 (a compromised value) and 43197 (the true thermodynamical density ratio at room temperature). The predicted cavitating flows are shown as Fig. 5. The steady attached sheet cavity is predicted with the default maximum density ratio of 1000 , but the vortex flow in the rear region is not observed. The numerical results predicted under the maximum density ratios of 20000 and 43197 also present the quasi-steady sheet cavity, with a clockwise vortex induced by the interaction of re-entrant jet and main flow at the cavity closure. Disturbed by the vortex, small bubble clusters shed from the rear part of the cavity, which is consistent with the experiment results.

Figure 6 compared the predicted pressure distributions along the suction surface of the hydrofoil with different maximum density ratios. As shown in Fig. 5 and 6 , the larger value of $\rho_{1} / \rho_{\mathrm{v}, \mathrm{clip}}$ enhances the mass transfer rate between the liquid and vapor phases, which results in a significant rise of the vapor fraction inside the cavity. The length of the attached cavity calculated with the default value of 1000 is almost $0.24 \mathrm{c}$ and it has a large perdition error compared with the experiment value of $0.4 \mathrm{c}$. However, with the increasing of maximum density ratio, the predicted cavity length raises accordingly. This augmentation almost reaches saturation when the maximum density ratio increases into about 20000. The predicted parameter is only slightly increased even when the ratio is as high as 43197 . The numerical results with the value of 20000 and 43197 correspond well to the reported experimental results.

\section{Effect of Maximum Density Ratio on Simulating Unsteady Cloud Cavitation}

When the cavitation number is reduced to 0.8 , the sheet cavitation grows and the trailing edge becomes 
increasingly unsteady. Accompanying this trend, bubbles are shed massively in the rear portion of the cavity and the cloud cavitation is formed. Figure 7 indicates the temporal evolutions of the cavity shapes around the hydrofoil under the cloud cavitation condition obtained via numerical methods with three maximum density ratios and experiment (Shi et al., 2012). In the simulation results, the vapor volume fraction was used to illustrate cavity shape.

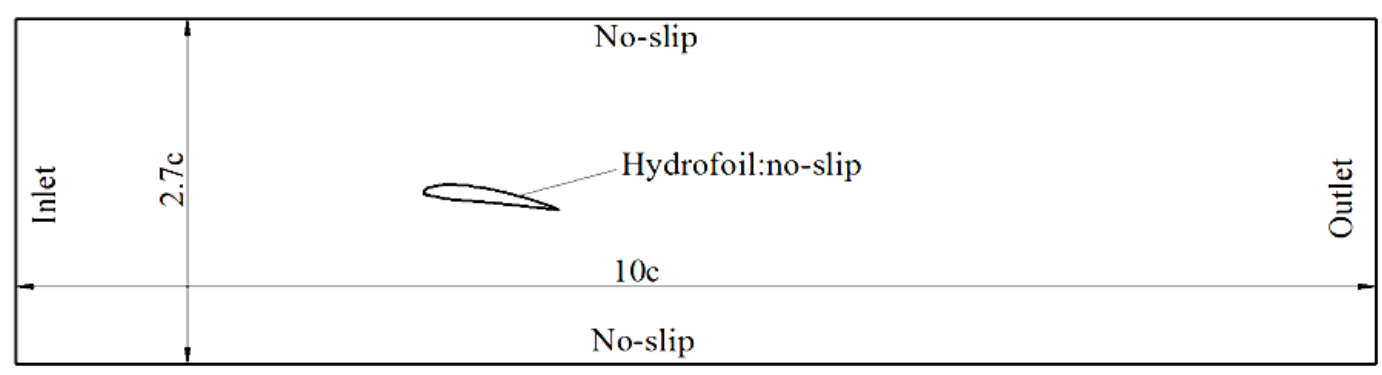

Fig. 1. Computational domain and boundary conditions

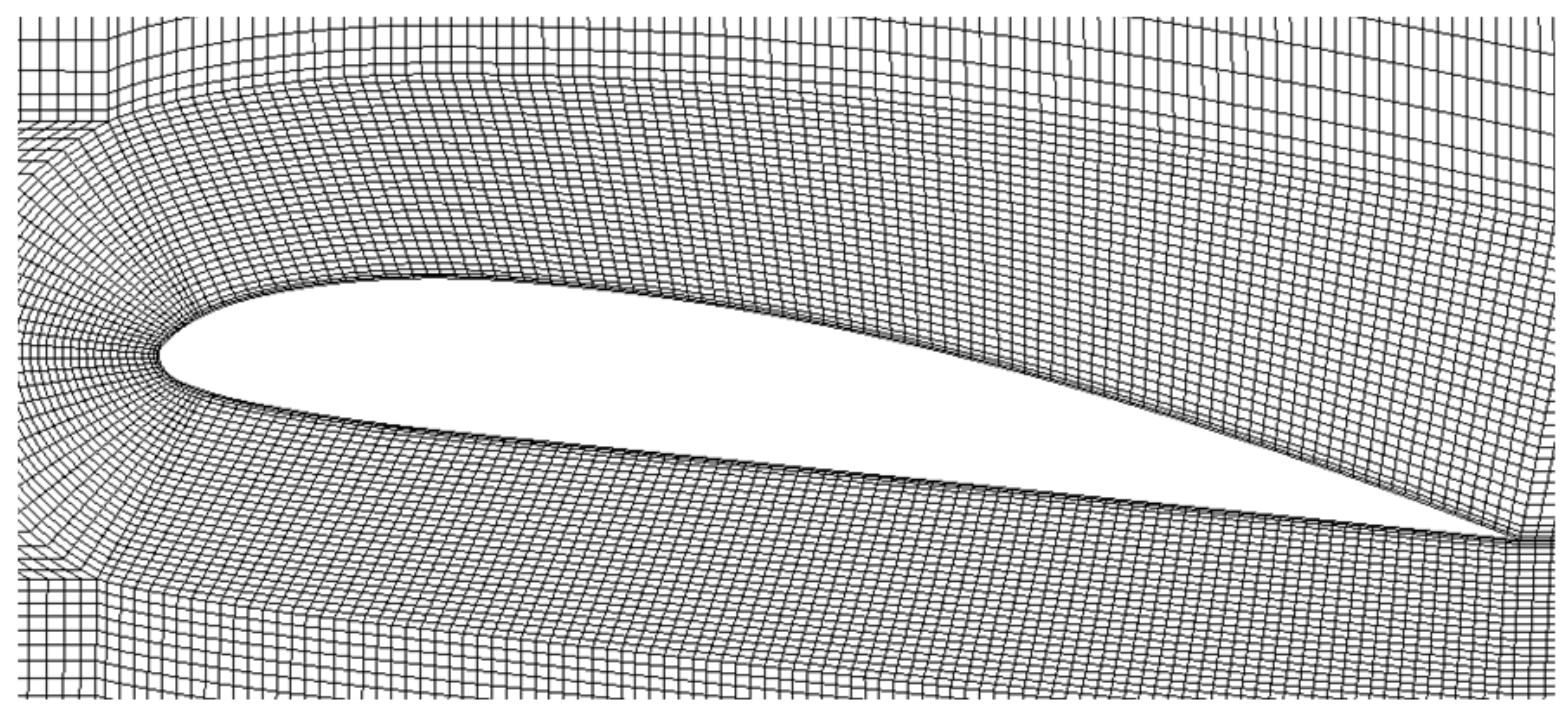

Fig. 2. Mesh near the hydrofoil

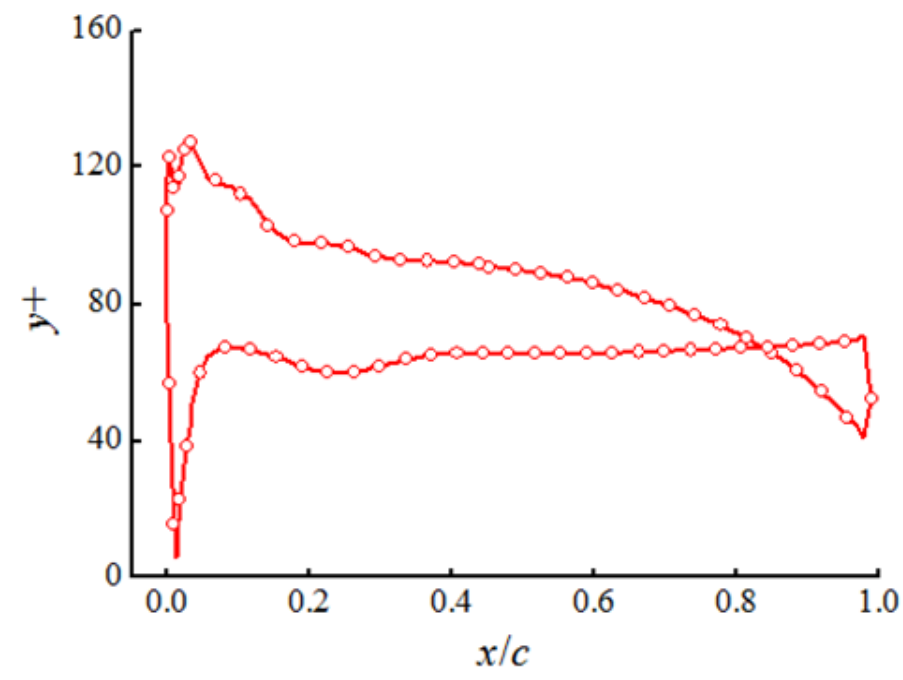

Fig. 3. Variation of $y+$ along the hydrofoil surface 


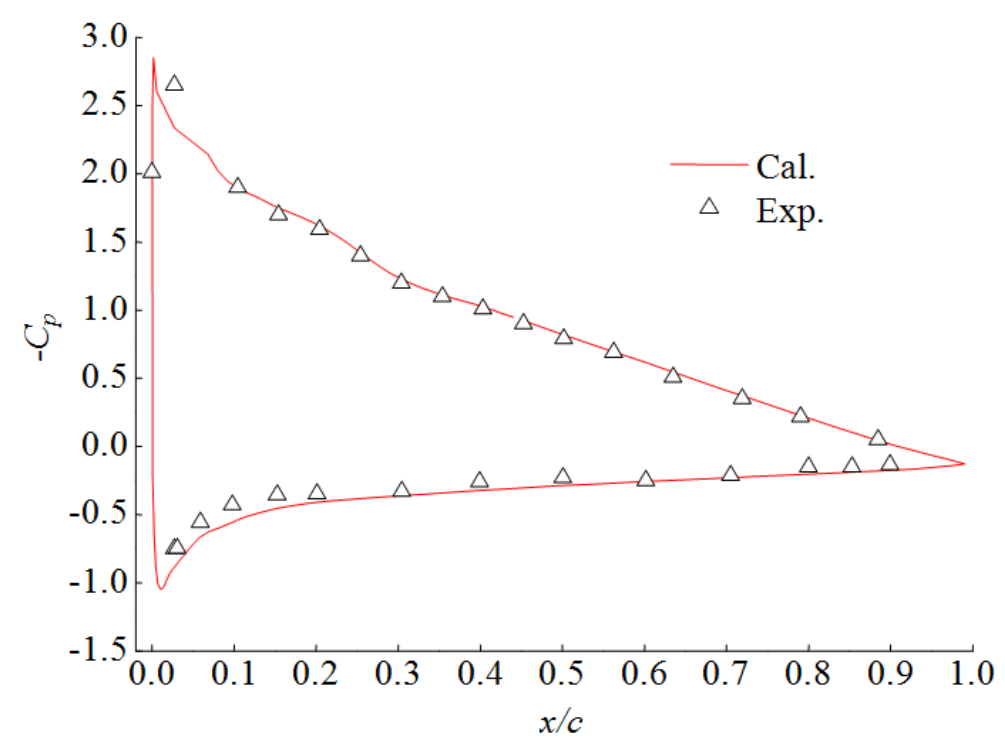

Fig. 4. Pressure coefficient distribution of Clark-Y hydrofoil, $U_{\text {in }}=10 \mathrm{~m} \mathrm{sec}^{-1}, \alpha=8^{\circ}$, non-cavitation

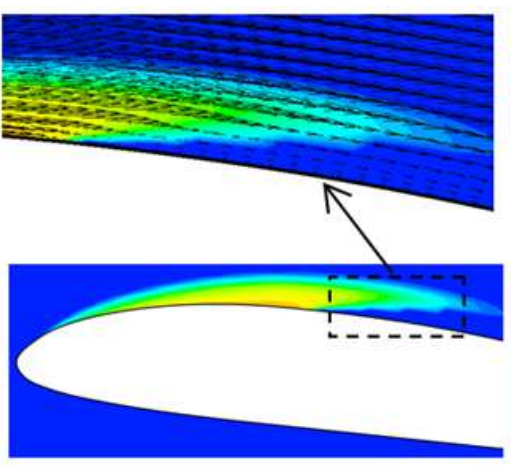

(a)

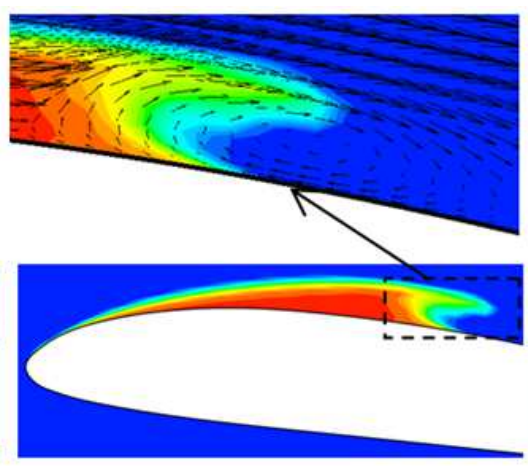

(b)

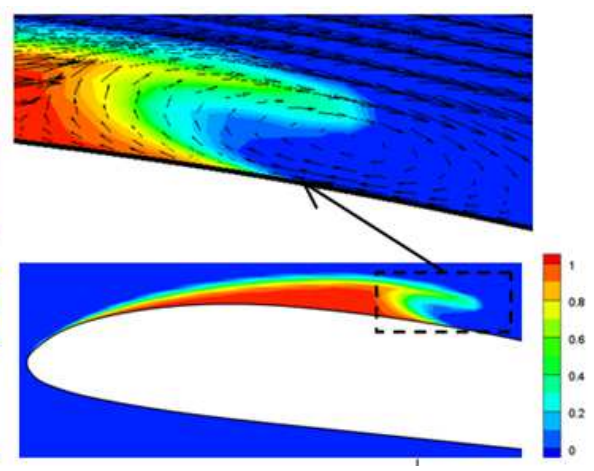

(c)

Fig. 5. Calculated cavity shape and velocity distribution with different $\rho_{l} / \rho_{v, \text { clip }}$ (a) $\rho_{l} / \rho_{v, \text { clip }}=1000$ (b) $\rho_{l} / \rho_{v, \text { clip }}=20000$ (c) $\rho_{l} / \rho_{v, \text { clip }}=43197$

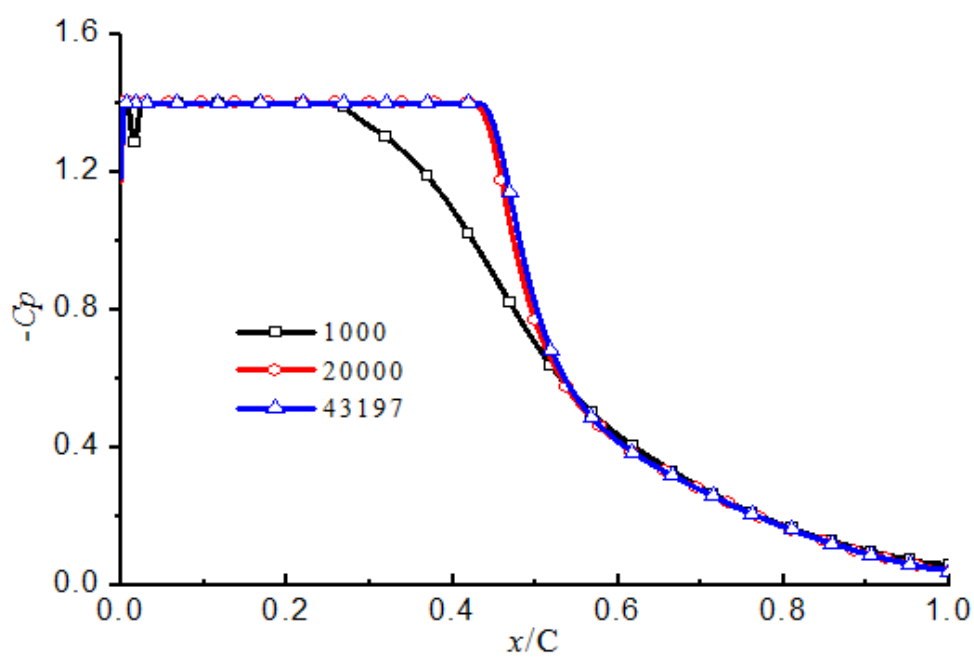

Fig. 6. Pressure distributions along the suction surface of the hydrofoil 


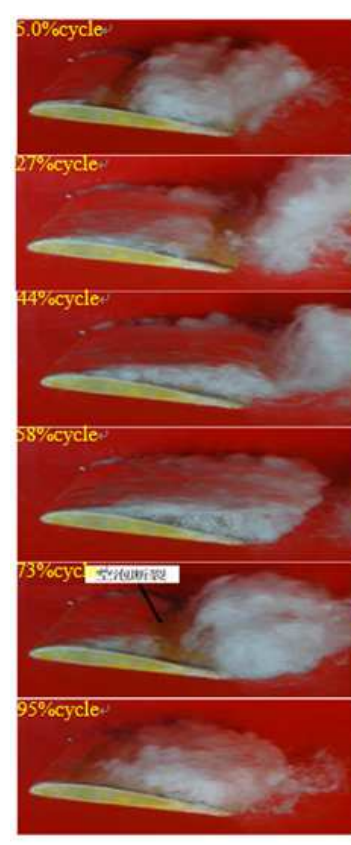

(a)

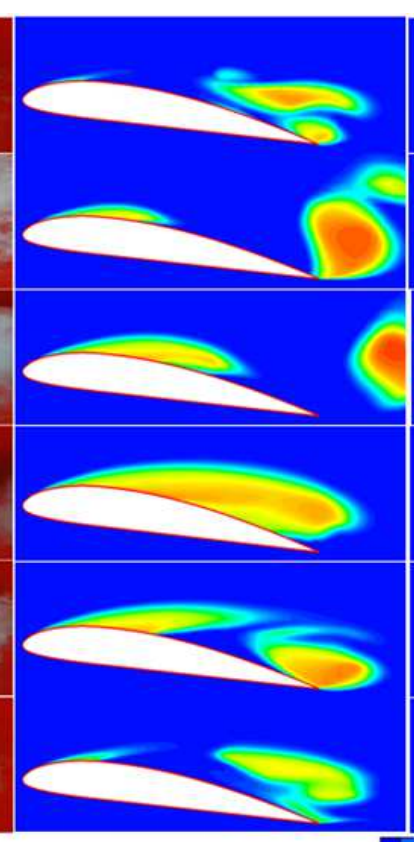

(b)

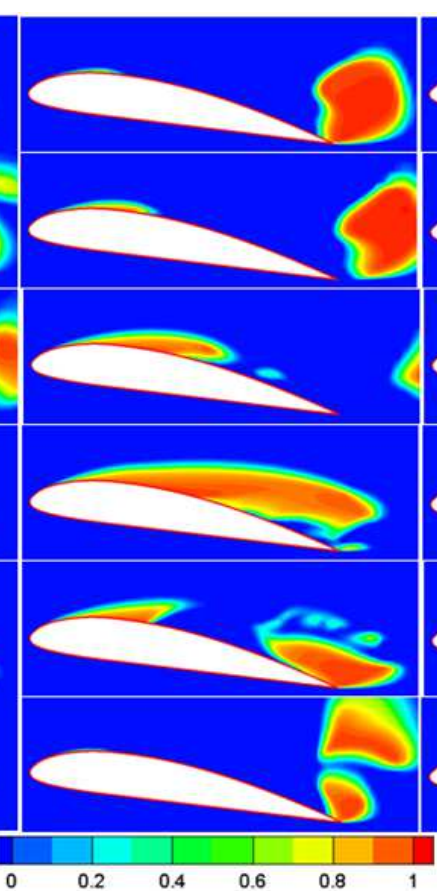

(c)

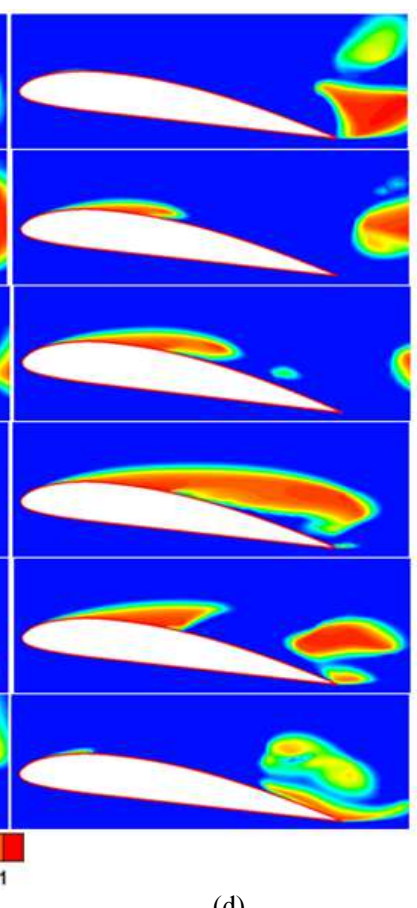

(d)

Fig. 7. Time evolution of cavity shape in the experiment and the calculation with different $\rho_{l} / \rho_{v, \text { clip }}$ (a) Experiment (b) $\rho_{l} / \rho_{v, \text { clip }}=1000$ (c) $\rho_{l} / \rho_{v, \text { clip }}=20000$ (d) $\rho_{l} / \rho_{v, \text { clip }}=43197$

As shown in Fig. 7, all the prediction with three ratios describe the quasi-periodic evolutions of cloud cavitation and the cavity shedding is observed which is consistent with the experiment. The cloud cavitation is a complicated process and can be divided into the following steps: (a) The thin attached sheet cavity is formed in the low-pressure region of hydrofoil and the cavity length and thickness increase over time. The shedding cloud cavity in the last cycle still remains in the trailing edge of the hydrofoil. (b) The attached sheet cavity grows slowly up to the maximum length at about $55 \%$ of the cycle time, while the cloud cavity in the trailing edge moves downstream and collapses in the downstream high-pressure zone. (c) The reentrant jet flowing upstream cuts the sheet cavity into two parts: Attached sheet cavity in the foreside of the cavity and shedding vortex structure in the rear region. (d) The attached sheet cavity shrinks sequentially towards the leading edge and the shedding vapor cloud is rolled up and convects downstream following the main flow. While the residual cavity grows again, another cycle begins.

The differences of predictions with three maximum density ratios mainly reflect in the vapor volume fraction and the smoothness of the interface between water and vapor. The vapor volume fraction in the attached sheet cavity and the shed cloud cavity is low, substantially below 0.8 , when simulated with the ratio of the default value 1000 . With the improvement of the maximum density ratio, the predicted vapor volume fraction increases significantly, especially in most region of cloud cavity, the value reaches above 0.95 . The predicted vapor volume fraction can reach a reasonable value via increasing the maximum density ratio. There are also some differences in the cavity contours predicted by the three maximum density ratios. The cavity contour predicted with the default value 1000 is clear and smooth, no small-scale bubbles shedding off. When the maximum density ratio increases to 20000 and 43197 , the increasing mass transfer rate makes the interface more unstable, accompanied a series of small-scale bubbles shedding intermittently, which is more consistent with the cavity shape in the experiment.

In order to show the effect of maximum density ratio clearly, the predicted transient evolution of the cavitating flow was illustrated through the time history of the total vapor volume, $V_{\text {cav }}$, where $V_{\text {cav }}$ is defined as:

$V_{c a v}=\sum_{i=1}^{n} \alpha_{i} V_{i}$

where, $n$ is the total number of control volumes in the computational domain, $\alpha_{i}$ the vapor volume fraction in each control volume and $V_{i}$ the volume of each cell.

As can be seen from Fig. 8, the time history of the total vapor volume is consistent with the transient evolution of cavity shape, taking on a quasi-periodic variation with time. With the increase in the maximum density ratio, the total vapor volume raises significantly. 


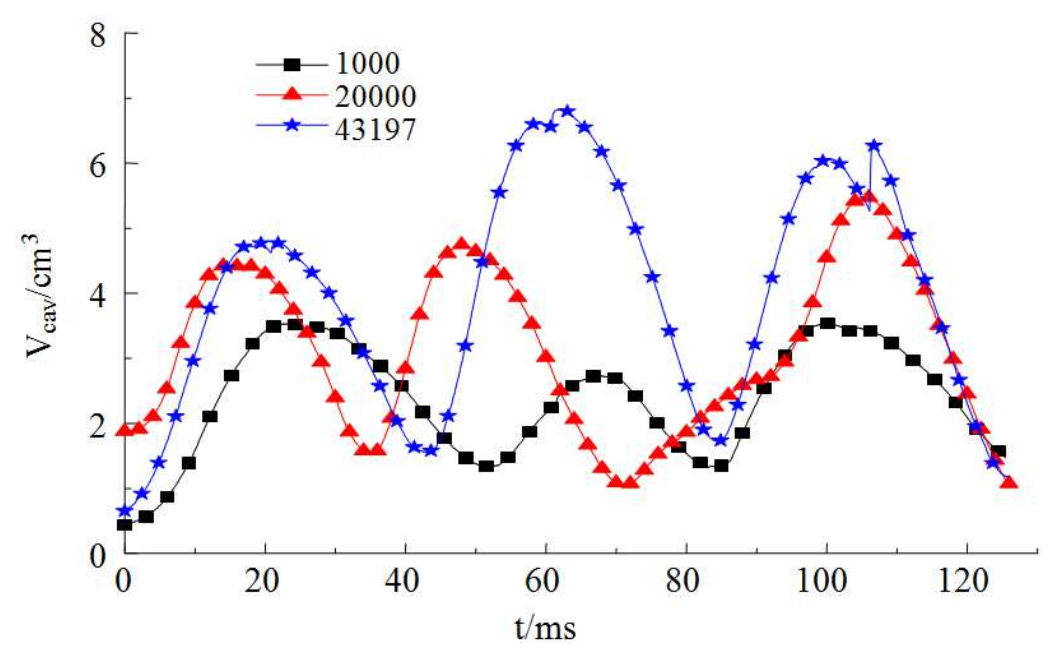

Fig. 8. Calculated total vapor volume with different $\rho_{l} / \rho_{v, c l i p}$

The predicted maximum total vapor volume by the value 43197 is about twice the amount by the default value 1000 , but has a slight difference with the amount by the value 20000 . It indicates that when the maximum density ratio increases to a certain extent, the total vapor volume doesn't change with maximum density ratio significantly.

In the present study, we find that the default $\rho_{l} / \rho_{v, \text { clip }}=1000$ ensures the numerical stability and accelerates the convergence of the calculation, but it underestimates the development of the cavitation. Considering the balance between the prediction accuracy and convergence, the suitable maximum density ratio of 20,000 is employed to reach an optimum between accuracy and convergence.

\section{Conclusion}

The FBM and a homogeneous cavitation model were employed to simulate cavitating flow around a 2D Clark-y hydrofoil. The effect of a maximum density ratio between liquid and vapor on sheet and cloud cavitating simulation was investigated. The conclusions are as follows:

- The maximum density ratio has a significant impact on cavitating simulation. The predicted cavitation with default value 1000 is underestimated compared with experiment. With the increasing of maximum density ratio, the interaction interface between liquid and vapor becomes unstable, accompanying the intermittent shedding of small-scale cavities. The cavity length and vapor volume fraction also increase

- When the maximum density ratio is increased to some degree, its effect on cavitation flow calculation becomes unobvious. A smaller maximum density ratio can ensure numerical stability but the result predicted with true density ratio is more accurate, so 20000 is recommended as the value of maximum density ratio in cavitation model to reach an optimum between accuracy and convergence

\section{Acknowledgement}

The researchers gratefully acknowledge the technical and financial support provided by Jiangsu University.

\section{Funding Information}

This work was financially supported by the National Natural Science Foundation of China (Grant No. 51109093).

\section{Author's Contributions}

Guangjian Zhang: Did data-analysis and wrote the manuscript.

Weidong Shi: Supplied the line of thought and organized the study.

Ling Zhou: Did the numerical simulating and collected data.

Desheng Zhang: Reviewed the whole paper critically.

\section{Ethics}

The authors confirm that this article is original and contains previously unpublished material. The corresponding author verifies that other authors have read and approved the manuscript and no ethical issues are violated. 


\section{References}

Ducoin, A., B. Huang and Y.L. Yong, 2012. Numerical modeling of unsteady cavitating flows around a stationary hydrofoil. Int. J. Rotat. Machinery, 2002: 215678-215624. DOI: 10.1155/2012/215678

Esfahanian, V., P. Akbarzadeh and K. Hejranfar, 2012. An improved progressive preconditioning method for steady non-cavitating and sheet-cavitating flows. Int. J. Numerical Meth. Fluids, 68: 210-232. DOI: $10.1002 /$ fld.2502

Huang, B., G. Wang and B. Zhang, 2009. Evaluation of the cavitation models on the numerical simulation of cloud cavitating flows around a hydrofoil. Trans. Beij. Instit. Technol., 29: 785-789.

Huang, B., G. Wang and H.T. Yuan, 2010. Analysis of the influence of experience constants in cavitation models for cavitation flow computations. Science, 3: 1430-1435.

Ji, B., X.W. Luo and Y.L. Wu, 2013. Numerical analysis of unsteady cavitating turbulent flow and shedding horse-shoe vortex structure around a twisted hydrofoil. Int. J. Multiphase Flow, 51: 33-43. DOI: 10.1016/j.ijmultiphaseflow.2012.11.008

Johansen, S.T., J.Y. Wu and W. Shyy, 2004. Filter-based unsteady RANS computations. Int. J. Heat Fluid Flow, 25: 10-21.

DOI: $10.1016 /$ j.ijheatfluidflow.2003.10.005

Kunz, F., B.W. Siebert and W.K. Cope, 1998. A coupled phasic exchange algorithm for three-dimensional multi-field analysis of heated flows with mass transfer. Comput. Fluids, 27: 741-768.

DOI: $10.1016 / \mathrm{S} 0045-7930(97) 00064-9$
Leroux, J.B., O. Coutier-Delgosha and J.A. Astolfi, 2005. A joint experimental and numerical study of mechanisms associated to instability of partial cavitation on two-dimensional hydrofoil. Phys. Fluids, 17: 052101-052120. DOI: $10.1063 / 1.1865692$

Morgut, M., E. Nobile and I. Biluš, 2011. Comparison of mass transfer models for the numerical prediction of sheet cavitation around a hydrofoil. Int. J. Multiphase Flow, 37: 620-626.

DOI: 10.1016/j.ijmultiphaseflow.2011.03.005

Shi, S.G., G.Y. Wang, H.T. Yuan and F. Wang, 2012. The investigation of unsteady cavitation flow structure around a three-dimensional hydrofoil by numerical and experimental methods. Eng. Mechan., 29: 346-353.

Singhal, A.K., M.M. Athavale, H.Y. Li and Y. Jiang, 2002. Mathematical basis and validation of the full cavitation model. J. Fluids Eng., 124: 617-624. DOI: $10.1115 / 1.1486223$

Wang, G.Y., I. Senocak, W. Shyy, T. Ikohagi and S.L. Cao, 2001. Dynamics of attached turbulent cavitating flows. Progress Aerospace Sci., 37: 551-581. DOI: $10.1016 / \mathrm{S} 0376-0421(01) 00014-8$

Zhou, L.J. and Z.W. Wang, 2008. Numerical simulation of cavitation around a hydrofoil and evaluation of a RNG k- $\varepsilon$ model. J. Fluids Eng., 130: 011302-011308. DOI: 10.1115/1.2816009

Zwart, P.J., A.G. Gerber and T. Belamri, 2004. A twophase flow model for predicting cavitation dynamics. Proceedings of the International Conference on Multiphase Flow, (CMF' 04), Yokohama, Japan. 